\title{
Correlación de Propiedades de Aceites Vegetales y Sebo Vacuno Usando Aproximantes de Padé
}

\author{
Mónica P. Guerrero ${ }^{1}$, Luis A. Toselli ${ }^{1}$ y José O. Valderrama ${ }^{2,3}$ \\ (1) Universidad Tecnológica Nacional, Facultad Regional Villa María, GISIQ, Av. Universidad 450 \\ X5900HLR Villa María (Córdoba)-Argentina (e-mail: mpguerrero@infovia.com.ar) \\ (2) Universidad de La Serena, Facultad de Ingeniería, Casilla 554, La Serena-Chile \\ (e-mail: jvalderr@userena.cl) \\ (3) Centro de Información Tecnológica, Casilla 724, La Serena-Chile
}

\begin{abstract}
Resumen
Se han utilizado los aproximantes de Padé para correlacionar algunas propiedades necesarias para el diseño y modelado de intercambiadores de calor de tubo y coraza usados en procesos de elaboración de aceites vegetales y sebo vacuno y en la producción de biodiesel, entre otras aplicaciones. Los aproximantes de Padé son funciones racionales que tienen la capacidad de representar funciones complejas con un número reducido de parámetros. Se ha considerado la densidad, el calor específico, la viscosidad y la conductividad térmica de estas sustancias y se han determinado los parámetros del modelo estudiado usando datos experimentales de estas propiedades. El modelo de Padé usado muestra ser adecuado para el cálculo de las propiedades estudiadas obteniendo desviaciones similares o inferiores a otros modelos altamente no lineales.
\end{abstract}

Palabras clave: aproximantes de Padé, aceites vegetales, sebo vacuno, biodiesel

\section{Correlation of Vegetable Oil and Beef Tallow Properties Using Padé Approximants}

\begin{abstract}
Padé approximants have been used to correlate some properties needed in the modeling and design of shell and tube heat exchangers used in the production of vegetables oils, beef tallow and biodiesel. Padé approximants are rational functions that have the capacity of representing complex functions with a reduce number of parameters. The properties considered were density, specific heat, viscosity and thermal conductivity of these substances and model parameters were determined using experimental data from the literature. The proposed general Padé model shows to be adequate to estimate the properties studied, obtaining deviations similar or lower than those of other highly non-linear models.
\end{abstract}

Keywords: Padé approximants, vegetable oils, beef tallow, biodiesel 


\section{INTRODUCCIÓN}

Existen numerosas aplicaciones en ingeniería que requieren de modelos matemáticos para representar ciertas variables de proceso o propiedades de las sustancias involucradas en estos. Propiedades físicas, termodinámicas y de transporte como densidad, capacidad calorífica, viscosidad y conductividad térmica son requeridas en diversos cálculos en ingeniería, tales como diseño de bombas, intercambiadores de calor, columnas de absorción, evaporadores, columnas de destilación, y secadores, entre otros (Lewis, 1987; Heldman y Lunn, 1992).

Los aceites vegetales y el sebo vacuno son ampliamente usados en la industria alimentaria, farmacéutica y cosmética (Hatti-Kaul et al., 2007; Dubois et al., 2007), aunque en los últimos años han tenido importantes aplicaciones en la fabricación de biodiesel (Van Gerpen, 2005; Knothe et al., 2005; Demirbas, 2007).

Aunque existen modelos teóricos y generalizados para estimar algunas propiedades de fluidos los modelos empíricos de tipo algebraico (polinomios, funciones exponenciales, funciones logarítmicas), son a veces necesarios para mayor exactitud. En estos modelos los parámetros del modelo algebraico son obtenidos mediante análisis de regresión de datos experimentales. En algunos casos específicos y con ciertas limitaciones, el modelo puede ser "linealizado" mediante redefinición de las variables involucradas en el modelo, pero en la mayoría de las situaciones dicha linealización no es posible, sin una sobre simplificación del problema. Para las propiedades consideradas en este trabajo existen diversos modelos en la literatura pero no han sido estudiados en forma rigurosa con un modelo simple y general como el que se presenta en este estudio (Lewis, 1987).

La aproximación de Padé es una herramienta útil en las matemáticas aplicadas y ha sido usada con frecuencia en problemas de mecánica estadística y termodinámica, pero no ha sido aplicado a la correlación de propiedades, como se propone en este estudio (Valderrama y Sandler, 1981; Baker y Graves- Morris, 1981). Sin embargo, pese al aparentemente rápido y sencillo tratamiento que permite la aproximación de Padé, se debe ser cuidadoso con algunos defectos matemáticos que tiene la aproximación, como lo discute Baker (2000). En el caso particular de las propiedades analizadas en este trabajo, estas pueden ser bien representadas mediante expresiones que cumplen con todas las propiedades de funciones "matemáticamente favorables" para el tipo de análisis desarrollado en este trabajo.

Los aproximantes de Padé son funciones racionales (un polinomio dividido por otro polinomio) que tienen la capacidad de representar funciones complejas con un número reducido de parámetros. Así, una función altamente no lineal que expandida en serie requeriría un gran número de términos puede ser representada con un aproximante de Padé simple de pocos coeficientes, para obtener el mismo grado de exactitud. Los aproximantes funcionan particularmente bien cuando se tienen series lentamente convergentes, pero son igualmente eficientes en otras situaciones (Baker, 2000). El aproximante de Padé se representa como $\mathrm{P}_{\mathrm{n}, \mathrm{m}}$ :

$P_{n, m}(x)=\frac{R_{m}(x)}{Q_{n}(x)}$

En esta ecuación, $R_{m}(x)$ y $Q_{n}(x)$ son polinomios de grado $m$ y $n$, respectivamente, siendo los aproximantes más utilizados aquellos en que el polinomio $R_{m}(x)$ tiene igual grado o sólo uno más que el polinomio $\mathrm{Q}_{\mathrm{n}}(\mathrm{x})$. Esto es $\mathrm{m}=\mathrm{n}+1$.

La figura 1 muestra como una función no lineal es representada por un polinomio de 5 términos (hasta $x^{4}$ ) y con una aproximación de Padé $(1,2)$. La representación de Padé es simplemente sorprendente hasta valores altos de $\mathrm{x}$. El porqué esto funciona así es aún un misterio para los matemáticos. Lo que si se sabe es que es difícil predecir de antemano que tan buena será una aproximación de Padé para una determinada aplicación y su exactitud depende de cada problema en particular. 


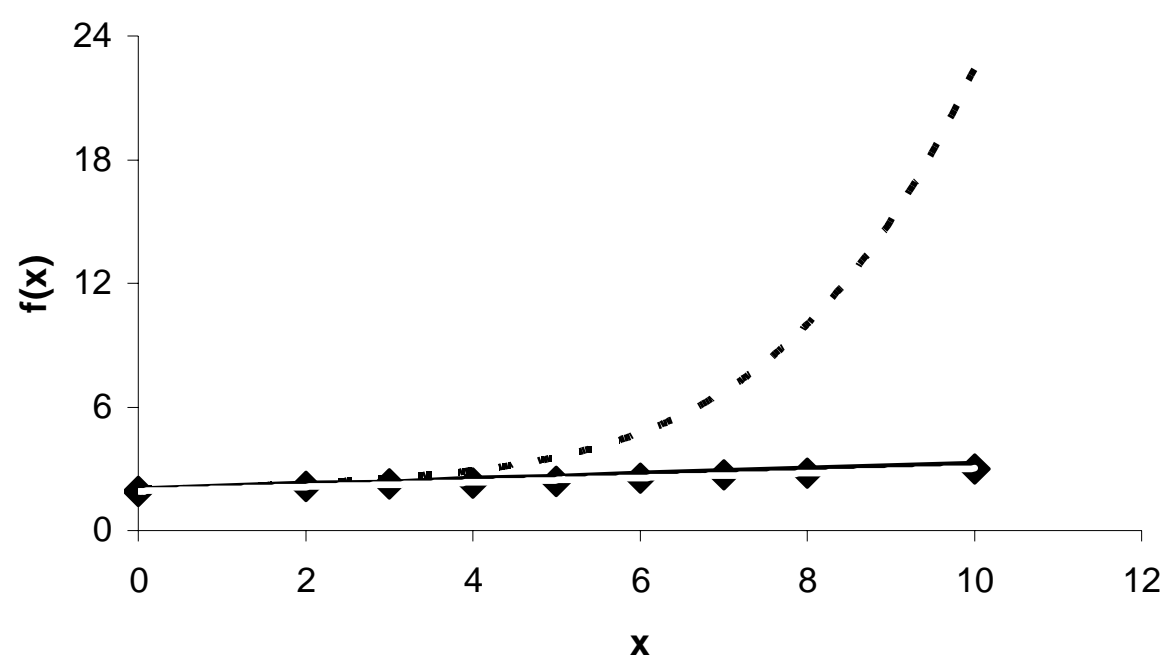

Fig. 1: Función $f(x)=\left[7+(1+x)^{4 / 3}\right]^{1 / 3}: \bullet$ la función exacta; modelada con una expansión en serie de 5 términos; _— aproximación de Padé $(1,2)$. Adaptado de Press et al. (1992).

\section{DESARROLLO DEL MODELO}

En este trabajo se usó el aproximante de Padé para modelar propiedades tales como la densidad, el calor específico, la viscosidad y la conductividad térmica de aceites vegetales y sebo vacuno. Se han usado datos experimentales de estas propiedades publicados en la literatura a temperaturas comprendidas entre 283 y 573 K (Karleskind, 1996; Erickson, 1990; Mohsenin, 1980), intervalos correspondientes al procesamiento industrial de estas sustancias

Aunque se exploró otras opciones, el aproximante de Padé $(1,1)$ resultó apropiado para representar las propiedades consideradas, dentro de los márgenes de error fijados. Si P representa a la propiedad (densidad, calor específico, viscosidad o conductividad térmica), y $\mathrm{T}$ la temperatura absoluta en Kelvin, la expresión evaluada fue:

$P_{1,1}(T)=\frac{a_{1}+b_{1} T}{d_{1}+c_{1} T}$

Esta expresión puede ser arreglada dividiendo por $\mathrm{d}_{1}$, para obtener un modelo de tres parámetros:

$P_{1,1}(T)=\frac{a+b T}{1+c T}$

En todos los casos estudiados se determinó la desviación media relativa $\Delta \mathrm{y} \%$ y la desviación media absoluta $|\Delta \mathrm{y} \%|$, como parámetros significativos de la exactitud del modelo (Valderrama y Álvarez, 2005). Estas desviaciones se definen de la siguiente forma:

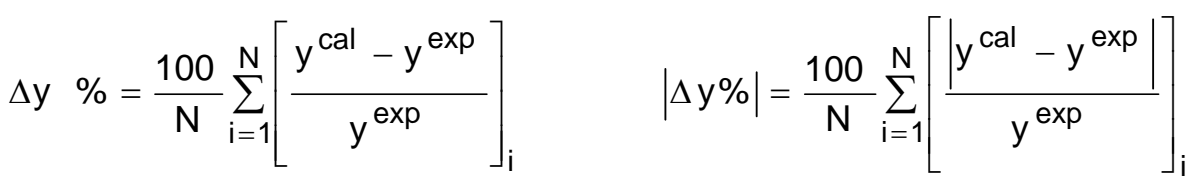


En estas ecuaciones solamente cuando $\mathrm{y}^{\exp }=0$, un caso de poco interés en este trabajo, se requiere de una definición distinta porque las ecuaciones (4) se hacen indeterminadas. De las definiciones anteriores la desviación relativa (sin valor absoluto) expresa claramente las desviaciones positivas y negativas reales con respecto a valores experimentales. Si la desviación relativa es cero o cercana a cero, significa que las desviaciones negativas y positivas, se cancelan, pero no indica nada sobre su magnitud. Si la desviación absoluta es pequeña, significa que las desviaciones son pequeñas pero no sabemos si son positivas o negativas. Ambos conceptos sin embargo determinan en forma aceptable la exactitud del modelo. Desde el punto de vista matemático, hay una serie de otros parámetros estadísticos que pueden ser de interés en algunas aplicaciones donde se requieren modelos robustos para extrapolaciones en amplios intervalos del valor de las variables. Desviación estándar, estadístico Z, sesgo, y curtosis son algunos parámetros que pueden ser usados para analizar las desviaciones entre los valores determinados por el modelo y los datos experimentales, determinar la robustez del modelo y garantizar que dicho modelo es estadísticamente aceptable; esto es que las desviaciones están normalmente distribuidas. Para conocer con detalle un conjunto de datos y lo que representa un determinado modelo, estos parámetros pueden ser de utilidad, en particular cuando se quiere evaluar o analizar varios conjuntos de datos. Sin embargo, las dos definiciones de desviación dadas en las ecuaciones (4) pueden ser suficientes para verificar la bondad del modelo, desde un punto de vista práctico.

La desviación estándar representa la dispersión de las variables y corresponde a una medida (cuadrática) de lo que se apartan los datos de su media. La normalidad puede evaluarse a través de pruebas estadísticas Z. Este parámetro está basado en el valor de la simetría y tamaño de la muestra. Si el valor calculado de $Z$ excede un valor crítico $( \pm 1,96)$, entonces la distribución es no normal. La curtosis (kurtosis) es una medida estadística que representa una característica del perfil de la distribución. Esta se refiere al "apuntamiento o llanura" de la distribución, comparada con la normal. La curtosis positiva indica una distribución relativamente apuntada, y la negativa indica una distribución relativamente achatada. En una distribución normal la curtosis es igual a 3, y a la diferencia entre ésta y los valores que resultan del modelo se denomina exceso de curtosis. Por otra parte el sesgo (skewness) es una medida estadística que describe la simetría de la distribución alrededor de un promedio. Si el sesgo es igual a cero, la distribución es simétrica; si el sesgo es positivo la distribución tendrá una cola asimétrica extendida hacia los valores positivos y viceversa si es negativo. Por lo tanto es deseable que la desviación estándar, el exceso de curtosis y el sesgo sean pequeñas, que la curtosis esté alrededor de 3.0, y que el estadístico $Z$ que determina la normalidad de la distribución de errores este entre -1.96 y +1.96.

\section{RESULTADOS Y DISCUSIÓN}

En la Tabla 1 se detallan las propiedades calculadas para los aceites vegetales y sebo vacuno, los parámetros correspondientes a las correlaciones obtenidas y los errores porcentuales promedios, relativos y absolutos. La Tabla 2 presenta un resumen de los estadísticos discutidos anteriormente obtenidos para las propiedades de aceites vegetales y sebo, a partir del modelo general planteado.

La Tabla 1 muestra que el aproximante de Padé $(1,1)$ proporciona un modelo de correlación muy ajustado a los datos experimentales, resultando desviaciones medias relativas y absolutas muy bajas para el calor específico y la densidad, similares a otros modelos aplicados en la literatura: del orden de $0.1 \%$ (Alvarado ,1996), 0,2 \% (Kubota et al., 1982), y 0,5 \% (Choi y Okos, 1986). Para el caso de la viscosidad, las desviaciones medias son un poco mayores (hasta 2.7\%), aunque estas son menores a las encontradas con modelos de tipo exponencial presentados en la literatura: $4.5 \%$ (Flores-Luque et al., 1982) y 3.6 \% (Viswanath y Natarajan, 1989). Analizando los estadísticos mostrados en la Tabla 2 puede observarse valores de desviación estándar pequeños, lo que indica una tendencia de concentración de datos cerca de la media, lo cuál es deseable. La escasa asimetría se pone de manifiesto en cada uno de los modelos analizados. Los valores de sesgo (curtosis) indican que la distribución presenta en la mayoría de los casos un leve achatamiento por debajo de la distribución normal, a excepción de un caso que supera levemente la misma. Por otra parte, el estadístico $Z$ dio lugar a valores que se mantienen dentro del intervalo esperado, que corresponde a un nivel de error del 0.05 , generando distribuciones próximas a la normal. 
Tabla 1: Parámetros de la ecuación (2) y desviaciones medias

\begin{tabular}{|c|l|r|r|c|c|c|}
\hline \multicolumn{1}{|c|}{ Propiedad } & & $a$ & $b$ & $c$ & $\% \Delta Y$ & $\% / \Delta Y /$ \\
\hline \multirow{5}{*}{ Densidad } & Girasol crudo & 933.77 & -0.0651 & $4.97 \mathrm{E}-11$ & $-1.18 \mathrm{E}-08$ & $4.50 \mathrm{E}-04$ \\
\cline { 2 - 7 } & Girasol refinado & 1171.66 & 0.3177 & $1.28 \mathrm{E}-03$ & $-4.45 \mathrm{E}-05$ & $5.25 \mathrm{E}-03$ \\
\cline { 2 - 7 } & Soja cruda & 934.93 & -0.0650 & $4.76 \mathrm{E}-11$ & $-1.15 \mathrm{E}-12$ & $3.02 \mathrm{E}-10$ \\
\cline { 2 - 7 } & Soja refinado & 933.62 & -0.0664 & $-1.01 \mathrm{E}-07$ & $-2.12 \mathrm{E}-13$ & $6.90 \mathrm{E}-07$ \\
\cline { 2 - 7 } & Sebo & 1185.32 & -0.8326 & $2.70 \mathrm{E}-12$ & $-1.03 \mathrm{E}-11$ & $2.32 \mathrm{E}-02$ \\
\hline \multirow{5}{*}{ Cond. Térmica } & Girasol Ref. & 0.179 & -0.0001 & $-8.65 \mathrm{E}-06$ & $-0.10 \mathrm{E}-03$ & $8.21 \mathrm{E}-02$ \\
\cline { 2 - 7 } & Sebo & 0.121 & -0.0004 & $-3.50 \mathrm{E}-03$ & $0.12 \mathrm{E}-01$ & $8.46 \mathrm{E}-01$ \\
\hline \multirow{5}{*}{ Calor Específico } & Girasol Ref. & 0.102 & 0.0012 & $2.14 \mathrm{E}-04$ & $-5.31 \mathrm{E}-05$ & $3.93 \mathrm{E}-02$ \\
\cline { 2 - 7 } & Sebo & 1.404 & 0.0015 & $-3.04 \mathrm{E}-04$ & $-7.71 \mathrm{E}-05$ & $7.67 \mathrm{E}-02$ \\
\hline Viscosidad & Girasol Ref. & -15.290 & 0.0307 & $-3.79 \mathrm{E}-03$ & $0.79 \mathrm{E}+00$ & $1.09 \mathrm{E}+00$ \\
\cline { 2 - 7 } & Soja Ref. & -6.052 & 0.0094 & $-6.13 \mathrm{E}-03$ & $-1.38 \mathrm{E}-03$ & $2.69 \mathrm{E}+00$ \\
\cline { 2 - 7 } & Sebo & -7.736 & 0.0163 & $-3.39 \mathrm{E}-03$ & $-1,39 \mathrm{E}+00$ & $7.66 \mathrm{E}-01$ \\
\hline
\end{tabular}

Tabla 2: Parámetros estadísticos a partir del modelo general (2)

\begin{tabular}{|l|c|c|c|c|c|}
\hline \multicolumn{1}{|c|}{ Propiedades } & Desv. Est. & Curtosis & Exceso de Curtosis & $Z$ & Asimetría \\
\hline Densidad Soja Ref. & 0.243 & 1.87 & -1.130 & -0.37 & -0.28 \\
\hline Densidad Girasol Crudo & 0.226 & 1.96 & -1.042 & -0.19 & -0.14 \\
\hline Densidad Girasol Ref. & 1.102 & 2.35 & -0.642 & 1.66 & -0.66 \\
\hline Densidad Sebo & 2.874 & 1.70 & -1.297 & 1.20 & -0.10 \\
\hline Cond. Térmica Sebo & 0.069 & 3.17 & 0.173 & 1.32 & 1.45 \\
\hline Cond. Térmica Girasol Ref. & 0.007 & 1.73 & -1.271 & -0.37 & -0.41 \\
\hline Calor esp. Girasol Ref. & 0.062 & 1.71 & -1.286 & 0.35 & 0.39 \\
\hline Calor esp. Sebo & 0.217 & 1.79 & -1.207 & 0.10 & 0.07 \\
\hline Viscosidad Girasol Ref. & 1.074 & 2.39 & -0.610 & 1.91 & 1.53 \\
\hline Viscosidad Soja Ref. & 0.890 & 2.42 & -0.579 & 0.13 & 0.06 \\
\hline Viscosidad Sebo & 0.503 & 2.34 & -0.654 & 1.65 & 0.69 \\
\hline
\end{tabular}

\section{CONCLUSIONES}

A partir de los resultados, se puede obtener las siguientes conclusiones principales: i) las desviaciones medias relativas y absolutas encontradas (Tabla 1) junto a los estadísticos determinados (Tabla 2) indican que el aproximante de Padé representa bien los datos experimentales y es estadísticamente consistente; ii) el análisis de los estadísticos y los bajos valores del parámetro "c" en el aproximante de la ecuación (3) indican que la mayoría de los modelos presentan una escasa no linealidad; y iii) si bien existen otras correlaciones que ajustan los datos experimentales, el aproximante de Padé $(1,1)$ representa un método alternativo nuevo para modelar propiedades de alimentos con excelentes resultados.

\section{AGRADECIMIENTOS}

MPG y LAT agradecen el apoyo de la Facultad Regional Villa María de la Universidad Tecnológica Nacional para la realización de este trabajo. JOV agradece a la Universidad de La Serena y al Centro de Información Tecnológica, por permanente apoyo a través de varios proyectos. 


\section{REFERENCIAS}

Alvarado, J. de D.; Principios de Ingeniería Aplicados a Alimentos, Sec. Gen. de la OEA, Prog. Nac. de Des. Cient. y Tecn., Proy. Nac. de Biotecnología y Tecnología de Alimentos, 124-147 (1996).

Baker Jr. G.A.; Defects and the Convergence of Padé Approximants, Acta Applicandae Mathematicae: 61(1-3) ,37-52 (2000).

Baker, Jr. G.A. y P. Graves-Morris; Padé approximants, Part I: Basic Theory, Encyclopedia of Mathematics and Its Applications, Addison-Wesley, Reading, MA-USA (1981).

Choi, Y. y M. Okos; Physical and Chemical Properties of Foods, ASAE Publicatión 9, American Society of Agricultural Engineers. St Joseph-USA, 35-77 (1986).

Demirbas, A.; Importance of Biodiesel as Transportation Fuel, Energy Policy: 35(9), 4661-4670 (2007).

Dubois V. y otros cuatro autores; Fatty Acid Profiles of 80 Vegetable Oils with Regard to Their Nutritional Potential, European Journal of Lipid Science and Technology: 109 (9), 710-732 (2007).

Erickson, R.D.; Oil Degumming y Soybean Lecithin. Handbook of Soy Processing and Utilization, American Soybean Association, USA, 131-143 (1990).

Flores Luque, V., M. Cabrera y C. Gómez Herrera: Variaciones de la Viscosidad y de la Densidad con la Temperatura en Aceites de Oliva, Girasol y Soja Españoles. Grasas y Aceites: 33(6), 334-339 (1982).

Hatti-Kaul Rajni y otros tres autores; Industrial Biotechnology For The Production Of Bio-Based Chemicals-A Cradle-to-Grave Perspective, Trends in Biotechnology (Review): 25 (3), 119-124 (2007).

Heldman, D y D. Lunn; Handbook of Food Engineering, Ed. Marcel Dekker Inc., New York-USA, 563615 (1992).

Karleskind, A.; Oils and Fats Manual, A Comprehensive Treatise: Properties, Production, Applications, Intercept Ltd. Pub., Andover-UK (1996).

Knothe, G.; J. Van Gerpen e I. KrahJürgen; The Biodiesel Handbook, The American Oil Chemists Society, Boulder-USA, 12-24 (2005).

Kubota, K. y otros cuatro autores; Study on the Viscosity and Density Equations Respected Temperature of Vegetable Oils and Salad and Frying Oils, Nippon Shokugin Kogyo Gakkaishi: 29 (4); 195-201 (1982).

Lewis, M.; Physical Properties of Food and Food Processing Systems, Ellis Horwood Pub., Chichester Inglaterra, pp.465 (1987).

Mohsenin, N.; Thermal Properties of Foods and Agricultural Materials, Gordon and Breach Science Publishers, New York, USA, 318-320 (1980).

Press, W.H. y otros tres autores; Padé Approximants, en Numerical Recipes in FORTRAN: The Art o, Scientific Computing, 2nd edition, cap 5.12, 194-197, Cambridge University Press, Cambridge (1992).

Viswanath, D.S. y G. Natarajan; Data Book on the Viscosity of Liquids, Hemisphere Publishing Corp., New York, USA, (1989).

Valderrama, J.O. y S.I. Sandler; Padé Approximants, the Second Virial Coefficient, and Perturbation Theorç, Chem. Phys. Letters: 84, 119-122 (1981).

Valderrama, J.O. y V.H. Alvarez; Correct way of Reporting Results when Modeling Supercritical Phase Equilibria using Equations of State, Can. J. Chem. Eng.: 83, 1-4 (2005).

Van Gerpen J.; Biodiesel Processing and Production, Fuel Processing Technology: 86 (10), 10971107 (2005). 